\title{
A New Epidemic Spreading Model to Predict the Spread and Accumulation of Corona Virus (COVID-19) Positive Cases as a Function of Time
}

\author{
Richard D. Sudduth*
}

Recently an article describing a new model to predict the dominant S shaped curve of the percolation threshold for electrical conducting composites was published by this author. This model was essentially the first to successfully address to whole concentration range for electrically conducting composites with the same model. Several possible applications where this new percolation threshold model might also be applicable were indicated in this article. One of these applications was the spread of disease in a population during a disease epidemic.

At this point, this new Epidemic Spreading Model has been successful in predicting the spread of the Corona Virus (COVID-19) in the United States from the beginning of the accumulation of positive cases on January 22, 2020 using Corona Virus (COVID-19) data collected by Johns Hopkins University. Interestingly, this model also appears to be able to separate the disease propagation from the disease mitigation.

This model has also been reasonably successful in predicting the spread of the Corona Virus (COVID-19) worldwide as well. In addition, when the model values for the magnitude of the separate populations were neutralized it was apparent that the growth of the epidemic in the USA was significantly greater than that experienced by the World data.

\section{Introduction}

While the Corona Virus (COVID-19) has only been on the world stage since approximately December 2019, modeling the spread of this virus epidemic has already been addressed by several authors [1-17]. The spread of positive cases for COVID-19 in the United States as accumulated by Johns Hopkins University [18] through April 27 is indicated in Fig. 1. The data in Fig. 1 can be characterized as being exponential in character and this property is reflected in most of the models used to describe the spread of the COVID-19 virus epidemic. The model developed by Fanelli and Piazza [1] characterized the cumulative confirmed infected people $(\mathrm{C})$ or positive cases for the Coronavirus COVID-19 into the following different components.

Materials Research and Processing, LLC, 3718 Dunlin Shore Court, Peachtree Corners, GA 30092, 470-767-8660

*Corresponding author:

E-mail: RichSudduth@Earthlink.Net

DOI: 10.5185/amlett.2021.071644

$$
\mathrm{C}=\mathrm{I}+\mathrm{R}+\mathrm{D}
$$

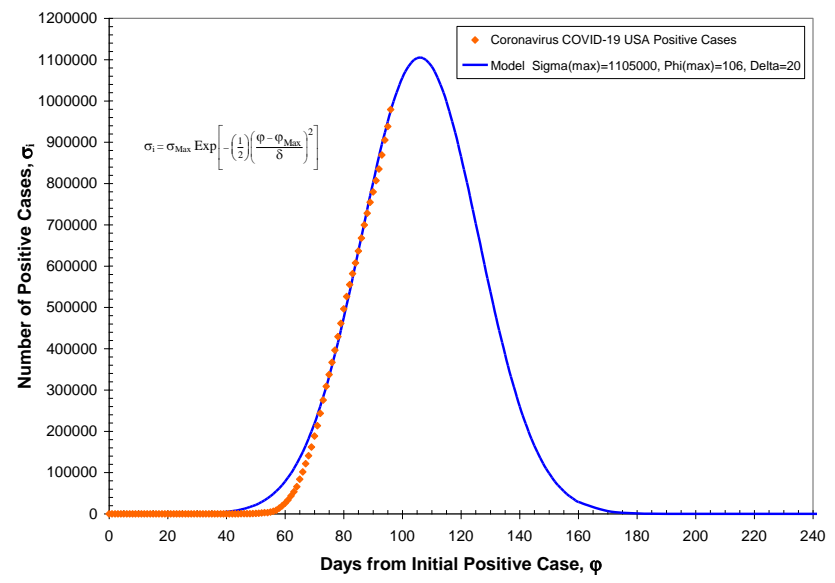

Fig. 1. Bell Shaped Model Addressing Positive Cases for Coronavirus COVID-19 in United States vs Days from Initial Positive Case.

The exponential model developed by Fanelli and Piazza [1] characterized equation 1 as model where the value of $\mathrm{C}$ rises to a plateau level. 
where

$\mathrm{C}=$ Cumulative confirmed infected people - at any point in time

I = Infected number of people - at any point in time

$\mathrm{R}=$ Recovered number of people

$\mathrm{D}=$ Deaths of people that have died from the virus

Equation 1 can also be rewritten as

$$
\mathrm{C}-\mathrm{R}=\mathrm{I}+\mathrm{D}
$$

One model that has been used to describe the spread of the Coronavirus would include the standard normal bellshaped curve that can be described as

$$
\sigma_{\mathrm{i}}=\sigma_{\mathrm{Max}} \operatorname{Exp}\left[-\left(\frac{1}{2}\right)\left(\frac{\varphi-\varphi_{\operatorname{Max}}}{\delta}\right)^{2}\right]
$$

where

$\sigma_{\mathrm{i}}=$ Accumulated Coronavirus COVID-19 positive cases

$\sigma_{\text {Max }}=$ Maximum Accumulated Coronavirus COVID-19 positive cases

$\delta=$ Standard Deviation

$\varphi=$ Number of days from the first Coronavirus COVID-19 positive case

$\varphi_{\text {Max }}=$ Number of days until the Maximum Coronavirus COVID-19 positive cases

The bell-shaped curve model shown in Fig. 1 was generated using the data that has been generated by Johns Hopkins University and published on their website [18]. While this Coronavirus epidemic did presumably start with $I_{0}=0, R_{0}=0, D_{0}=0$, it is clear that the bell-shaped curve in Fig. 1 cannot effectively describe the spread of this virus. Unfortunately, those who have died or who have become permanently infected by the virus can not be accounted for with the bell-shaped curve. When the COVID-19 epidemic is finally under control it is virtually certain that a significant number of people will not be able to recover.

\section{Development of a new model to describe epidemic spreading}

Recently an article [19] describing a new model to predict the dominant $\mathrm{S}$ shaped curve of the percolation threshold for electrical conducting composites was published by this author. Several possible applications where this new percolation threshold model might also be applicable were indicated in this article. One of these applications was the spread of disease in a population during an epidemic. The new percolation threshold model previously published by this author has been redefined to describe the spread of disease during an epidemic as

$$
\begin{gathered}
\left(\sigma_{\mathrm{i}} / \sigma_{\mathrm{o}}\right)=\left(\sigma_{\mathrm{F}} / \sigma_{\mathrm{o}}\right) \mathrm{F}(\varphi) \\
\mathrm{F}(\varphi)=\left(\frac{\varphi}{\delta}\right) \frac{\beta}{\varphi^{\alpha}}
\end{gathered}
$$

where

$$
\begin{aligned}
& \alpha=\text { Mitigation Effectiveness } \\
& \beta=\text { Mitigation Magnitude } \\
& \delta=\text { Threshold Upper Limit }
\end{aligned}
$$

$\sigma_{\mathrm{o}}=$ Initial Positive Coronavirus Cases

$\sigma_{F}=$ Final Positive Coronavirus Cases

$\varphi=$ Days since start of positive Cases

$\sigma_{\mathrm{i}}=$ Accumulated Positive Corona Virus Cases

$\mathrm{F}(\varphi)=$ Spreading Function

If the initial cases are assumed to be $\sigma_{o}=1$ then equations 5 and 6 can be combined to give

$$
\sigma_{i}=\sigma_{F}\left(\frac{\varphi}{\delta}\right) \frac{\beta}{\varphi^{\alpha}}
$$

The results in Fig. 2 have been generated again using the same data from Johns Hopkins University that were indicated in Figure 1. Note that a straight line through the maximum slope yields an intercept that can be interpreted as the critical day where the epidemic begins to go unstable. In this particular instance this percolation threshold was $\varphi_{c}=65$ days (or $3 / 26 / 20$ ) from the initial positive case. Therefore, if effective testing and tracing could have been accomplished in the first 65 days then the pandemic could possibly have been more easily avoided and the epidemic could have potentially been much easier to control.

In general, it has also been found that three concurrent mathematical conditions have been found to occur at the same curve location for S-shaped curves with percolation thresholds. In particular, the maximum slope occurs at the first inflection point on the curve and the maximum slope at that same location is then used to develop a straight line to calculate the maximum extrapolated percolation threshold on the $\mathrm{x}$-axis.

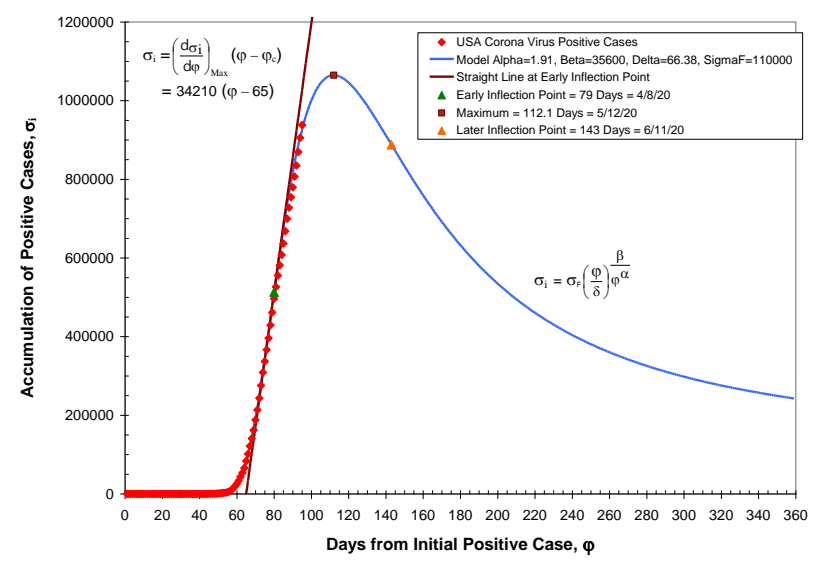

Fig. 2. New Epidemic Spreading Model Addressing Accumulative Corona Virus Positive Cases vs Days from Initial Positive Case in United States as of April 27, 2020.

Mathematically the inflection point in Fig. 2 was generated by setting the second derivative of equation (7) equal to zero. The second derivative of equation (7) has been generated in the appendix as equation (A-9). The location of the inflection points as indicated in the appendix must be solved by trial and error using the following equations.

$$
\left(z^{2}\left(\frac{\beta}{(\varphi)^{\alpha}}\right)-z(\alpha+1)-\alpha\right)=0
$$


where

$$
\mathrm{Z}=\left(1-\alpha \operatorname{Ln}\left(\frac{\varphi}{\delta}\right)\right)
$$

For the results in Fig. 2 the early inflection point occurred at $\varphi_{\text {EIP }}=79$ days (or 4/8/20).

As indicated in Fig. 2, once the early inflection point, $\varphi_{\text {EIP }}$, has been established then the maximum slope can be obtained at that point and the following equation can be used to obtain the maximum projected percolation threshold, $\left(\varphi_{\mathrm{ci}}\right)_{\max }=\varphi_{\mathrm{c}}$.

$$
\sigma_{\mathrm{EIP}}=\left(\frac{d_{\sigma i}}{d \varphi}\right)_{\text {Max }}\left(\varphi_{\mathrm{EIP}}-\varphi_{c}\right)
$$

The extrapolated maximum percolation threshold, $\varphi_{\mathrm{c}}$, shown in Fig. 2 can also evaluated using a different approach. The following equation as described in Appendix $\mathrm{B}$ allows the evaluation of the extrapolation that can be evaluated by trial and error to determine the maximum percolation threshold.

$$
\varphi_{\mathrm{ci}}=\varphi-\left(\frac{1}{\beta}\right)\left(\frac{\varphi^{\alpha+1}}{1-\alpha \operatorname{Ln}\left(\frac{\varphi}{\delta}\right)}\right)
$$

maximum percolation threshold can be established by plotting several projected percolation thresholds, $\varphi_{\mathrm{ci}}$, using equation (11) or (B-4) in Appendix B to obtain the maximum projected percolation threshold, $\left(\varphi_{\mathrm{ci}}\right)_{\max }=\varphi_{\mathrm{c}}$.

Note that several other calculated points are indicated on the curve in Figure 2 as summarized in Table 1. For reference it was found that the number of days, $\varphi_{\operatorname{Max}}$, for which equation (7) is a maximum can be easily generated as indicated in the Appendix A. The first derivative of equation (7) generated in the appendix yielded equation (A-3) which when set to zero and solved yielded the maximum accumulated positive cases, $\sigma_{\text {Max }}$, at:

$$
\varphi \operatorname{Max}=\delta \mathrm{e}^{(1 / \alpha)}
$$

The maximum, $\varphi_{\max }$, calculated from the results in Fig. 2 yielded $\varphi_{\max }=122.2$ days (or 5/12/20). This maximum has been indicated in Table $\mathbf{1}$ along with several other calculated values from Figure 2.

Table 1. USA and World Data for Positive Cases of Corona Virus (As of April 27, 2020 - Johns Hopkins University Website).

\begin{tabular}{lcc}
\hline & $\begin{array}{c}\text { Corona Virus } \\
\text { Model for USA }\end{array}$ & $\begin{array}{c}\text { Corona Virus } \\
\text { Model for World }\end{array}$ \\
\hline Mitigation Effectiveness, $\alpha$ & 1.910 & 1.843 \\
Mitigation Magnitude, $\mathrm{b}$ & 35600 & 15700 \\
Threshold Upper Limit, $\delta$ & 66.4 & 71.0 \\
Final Positive Cases, $\sigma_{\mathrm{F}}(\mathrm{Limit})$ & 110,000 & 990,000 \\
$\begin{array}{l}\text { Days to Percolation } \\
\text { Threshold, } \varphi_{\mathrm{c}}\end{array}$ & $65(3 / 26 / 20)$ & $59.8(3 / 20 / 20)$ \\
$\begin{array}{l}\text { Days to Early Inflection } \\
\text { Point, } \varphi_{\mathrm{EIP}}\end{array}$ & $79(4 / 8 / 20)$ & $75(4 / 4 / 20)$ \\
$\begin{array}{l}\text { Days to Maximum Positive } \\
\text { Cases, } \varphi \text { Max }\end{array}$ & $112.1(5 / 12 / 20)$ & $122.2(5 / 21 / 20)$ \\
$\begin{array}{l}\text { Days to Later Inflection } \\
\text { Point, } \varphi \text { LIP }\end{array}$ & $143(6 / 11 / 20)$ & $163(7 / 1 / 20)$ \\
Maximum Slope, $\left(\mathrm{d} \sigma_{\mathrm{i}} / \mathrm{d} \varphi\right)_{\text {Max }}$ & 34,210 & \\
Positive Cases, $\sigma_{\mathrm{i}}$, at $\varphi_{\mathrm{Max}}$ & $1,064,763$ & $3,333,175$ \\
\hline
\end{tabular}

\begin{tabular}{lcc}
\hline Positive Cases, $\sigma_{i}$ at 360 Days & 242,089 & $1,624,947$ \\
\hline Limit Considerations for the Spreading Function, $\mathbf{F}(\boldsymbol{\varphi})$
\end{tabular}

At this point Spreading Function, $F(\varphi)$, described by Equation (6) can be written as

$$
\mathrm{F}(\varphi)=\left(\frac{\varphi}{\delta}\right)^{\frac{\beta}{\varphi^{\alpha}}}
$$

If $\alpha>0, \beta>0$ and $\delta>0$ then the function $\mathrm{F}(\varphi)$ has the following interesting but important limits:

$$
\begin{gathered}
\varphi \rightarrow 0 \text { then } \mathrm{F}(\varphi) \rightarrow 0 \\
\varphi=\delta \text { then } \mathrm{F}(\varphi)=1.0 \\
\varphi=\varphi_{\max }=\delta \mathrm{e}^{(1 / \alpha)} \text { then } \mathrm{F}\left(\varphi_{\max }\right)=\left(\mathrm{e}^{1 / \alpha}\right)^{\beta / \mathrm{e} \delta^{\alpha} \geq 1} \\
\varphi \rightarrow \infty \text { then } \mathrm{F}(\varphi) \rightarrow 1.0
\end{gathered}
$$

In addition, when $\alpha>0, \beta>0$ and $\delta>0$ then the limits for equation (7) corresponding to the limits (13) to (16) are:

$$
\begin{gathered}
\varphi=0 \text { then } \sigma_{i}=0 \\
\varphi=\delta \text { then } \sigma_{i}=\sigma F \\
\varphi=\varphi_{\max } \text { then }\left(\sigma_{i}\right)_{\max }=\sigma F\left(\mathrm{e}^{1 / \alpha}\right)^{\beta / \mathrm{e}} \delta^{\alpha} \\
\varphi \rightarrow \infty \text { then } \sigma_{i}=\sigma F
\end{gathered}
$$

Consequently, the new model given by equation (7) then describes the spread of disease during an epidemic that begins at $\varphi=0$ where $\mathrm{F}(\varphi)=0$ and $\sigma_{i}=0$. However as $\varphi \rightarrow \infty$ then $\mathrm{F}(\varphi) \rightarrow 1.0$ and $\sigma_{\mathrm{i}} \rightarrow \sigma_{\mathrm{F}}$ which yields a limit that is not zero and is not negligible.

\section{Separation of the spreading function into separate mitigation and propagation components}

At this point it is useful to note that Spreading Function, $F(\varphi)$, described by equation (6) can be separated into a propagation component and an mitigation component where:

$$
\mathrm{F}(\varphi)=\left(\frac{\varphi}{\delta}\right)^{\frac{\beta}{\varphi^{\alpha}}}
$$

The propagation component can be described as

$$
\mathrm{F}_{\text {Propagation }}(\varphi)=\left(\frac{\varphi}{\delta}\right)
$$

And the mitigation component can be described as

$$
\mathrm{F}_{\text {Mitigation }}(\varphi)=\frac{\beta}{(\varphi)^{\alpha}}
$$

These three functions are plotted in Fig. 3 with the constants $\alpha, \beta$, and $\delta$ set at the same values as the results plotted in Figure 2.

Note in Fig. 3 that the Propagation Component, $\mathrm{F}_{\text {Propagation }}(\varphi)$, is a straight line with slope $(1 / \delta)$ that increases linearly with the number of days, $\varphi$, since the first positive case. However, the Mitigation Component, $\mathrm{F}_{\text {Mitigation }}(\varphi)$, decreases exponentially with an increase in the number of days, $\varphi$, since the first positive case. Note that as long as the number of days, $\varphi$, are less than the number of days as indicated by the Threshold Upper Limit, $\delta$, then the Propagation Component will be a fraction less 


\section{Advanced Materials Letters www.vbripress.com/aml}

than 1. This means that as long as the Mitigation Component, $\mathrm{F}_{\text {Mitigation }}(\varphi)$, is a large number as well as an exponent for the Propagation Component, F Propagation $(\varphi)$, then the combination described as function $F(\varphi)$ will generate a very small number essentially approaching zero when $\varphi$ is less than $\delta$. However, once the number of days, $\varphi$, exceeds the value of $\delta$ then the Propagation Component, Fropagation $(\varphi)$, will no longer be a fraction less than 1 and the Propagation Component will continue to increase significantly while the Mitigation Component will more rapidly approach zero forcing the combination of the two to cause the function $F(\varphi)$ to approach the value of one.

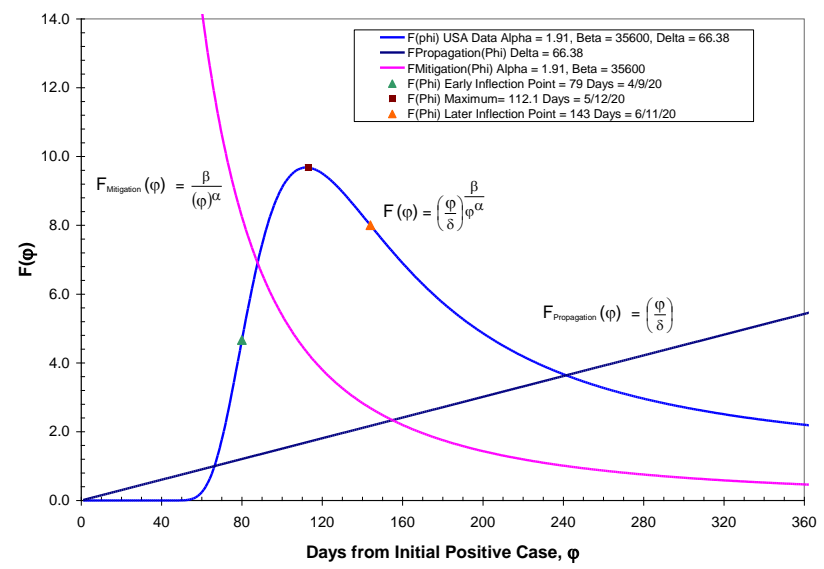

Fig. 3. Separation of Spreading Function, $F(\varphi)$, components into Mitigation and Propagation Components for USA Data as of April 27, 2020.

\section{Corona Virus (COVID-19) data comparison between the World and the United States}

The Corona Virus (COVID-19) data used in this study for both the USA and the World were collected and reported by Johns Hopkins University on their website [18]. However, only the data between January 22 and April 27 were utilized in this study. Since there seemed to be a general disregard for "Social Distancing" after April 27, it was felt that the best data for evaluating the model introduced in this study occurred in that time frame.

The model results indicated in Fig. 4 and Fig. 5 were generated from the worldwide Corona Virus (COVID-19) data supplied by Johns Hopkins University. The results for Fig. 4 and Fig. 5 have been summarized in Table 1 along with the calculated results from Figures 2 and 3 for the USA Corona Virus data.

Some important observations involved in the comparison of these two Corona Virus pandemic data sets as summarized in Table 1would include:

- While the USA data only applied to one country the World data eventually applied to over 100 countries that appeared to have the initiation of the Corona Virus (COVID-19) starting at different times. This partially accounts for why the model did not give a good fit for the initial World data as indicated in Figure 4 compared to the results in Figure 2.

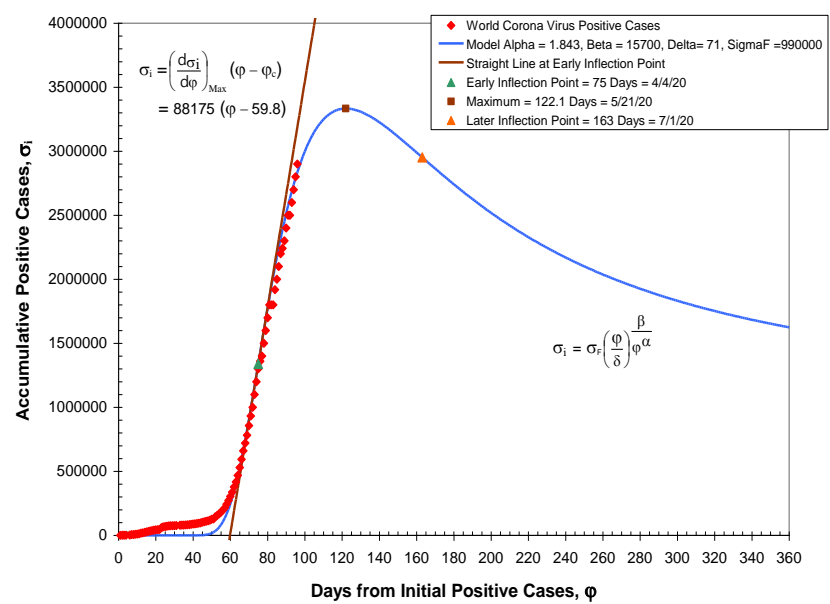

Fig. 4. New Epidemic Spreading Model Addressing Accumulative Corona Positive Cases vs Days from Beginning of Positive Cases in World as of April 27, 2020.

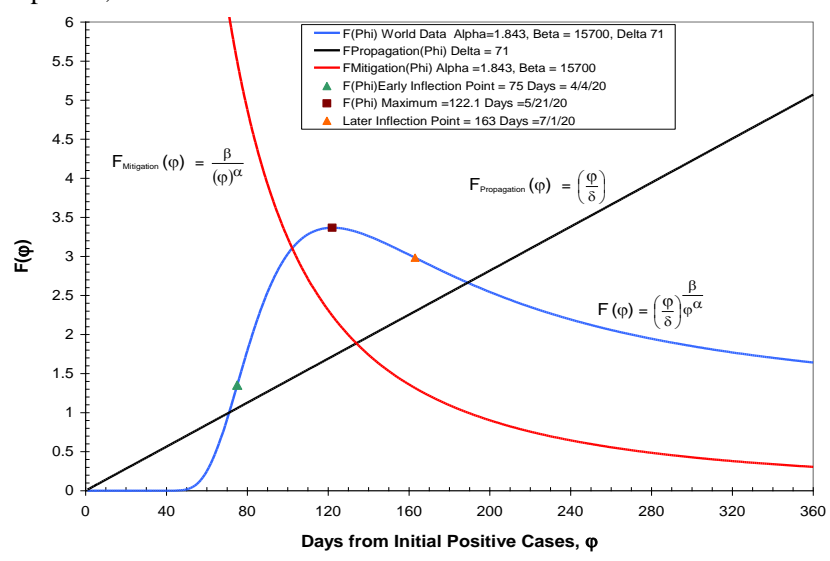

Fig. 5. Separation of Spreading Function, $F(\varphi)$, components into Mitigation and Propagation Components for World as of April 27, 2020.

The percolation thresholds for both the USA and World data are less than their respective values of, $\delta$, (Threshold Upper Limit) as expected.

- However, the percolation threshold for the World data was smaller and thus suggesting that the world data started earlier than the corresponding values for the USA data.

- It is particularly important to note that the slopes at the early inflection points were calculated to be greater for the World data than for the USA data as indicated in Table 1.

Interestingly the USA was expected to reach a maximum of 1,064,763 positive cases while the World was expected to reach a maximum of $3,333,182$ positive cases. In addition, both sets of data were expected to reach a maximum in the middle of May if everyone had been observing effective "Social Distancing".

After 360 days the results in Table 1 predict that the USA will still have 242,089 infected people or people 
that have died compared to a predicted 1,624,947 such people for the World data.

- Since more people are being addressed in the World data than the USA then maximum positive cases at, $\varphi_{\mathrm{Max}}$, and the final positive cases, $\sigma \mathrm{F}$, would be expected to be larger for the World data.

Initially it did appear that it would be difficult to compare the World and USA Corona Virus (COVID-19) data directly as indicated in the above results. However, it was found that these data sets are really much easier to compare directly using their $F(\varphi)$ components as indicated in the next section. This then resulted in completely new insights into the comparison of these data sets.

\section{Corona Virus (COVID-19) $\mathbf{F}(\varphi)$ components comparison between the World and the United States}

By dividing by the magnitude of their Final projected Positive Corona Virus (COVID-19) Cases, $\sigma F$, it was found that the USA and the World Corona Virus data results could be compared directly using their $\mathrm{F}(\varphi)$ components. A direct comparison of the $F(\varphi)$ components for both the USA and the World Corona Virus data sets was found to yield significant new insights into the comparison of these data sets. This elucidation is readily apparent in Table 2 that addresses the results as related to $F(\varphi)$ for the World and USA Corona Virus data sets. Comparisons of the components of $F(\varphi)$ in Fig. 6, Fig. 7 and Fig. 8 provide a direct comparison of the mitigation, $\mathrm{F}_{\text {Mitigation }}(\varphi)$, propagation, $F_{\text {Propagation }}(\varphi)$, and overall $F(\varphi)$ values respectively for the USA vs the World Corona Virus (COVID-19) data sets.

Some important observations involved in the comparison of these two Corona Virus pandemic data sets would include:

- Comparison of the results in Fig. 6 appears to indicate that the Mitigation process was apparently somewhat more effective in the USA than in the World.

- The Propagation process of the virus was also greater in the USA data compared to the World data as indicated in Fig. 7. These counteracting results did not directly clarify which component was more dominant.

- However, after the magnitude of their separate populations had been neutralized it was apparent in Figure 8 that the growth of the epidemic in the USA was significantly greater than that experienced by the World data.

- Table 2 also indicates that the maximum Corona Virus $F(\varphi)$ growth rate at their initial inflection points was 3.5 times greater for the USA resultant slope (0.311) compared to the slope (.0891) for World data after their separate populations had been neutralized

- In addition, Table 2 indicates that the maximum $\mathrm{F}(\varphi)$ value was found to be 2.9 times greater for the USA data (9.68) than that found for the World data (3.37).

- Finally, after 360 days the $\mathrm{F}(\varphi)$ results in Table 2 indicate that the USA will still have a higher relative population infected by the Corona Virus (2.2) compared to the relative infected World population (1.64).

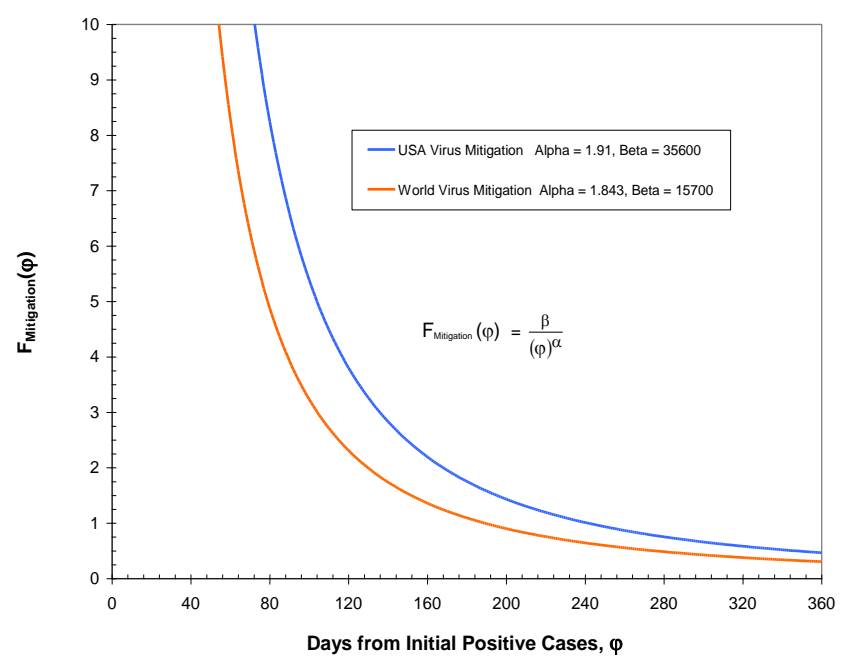

Fig. 6. Spreading Function, $F(\varphi)$, Comparison of Mitigation Components $\mathrm{F}_{\text {Mitigation }}(\varphi)$ for USA and World Data as of April 27, 2020.

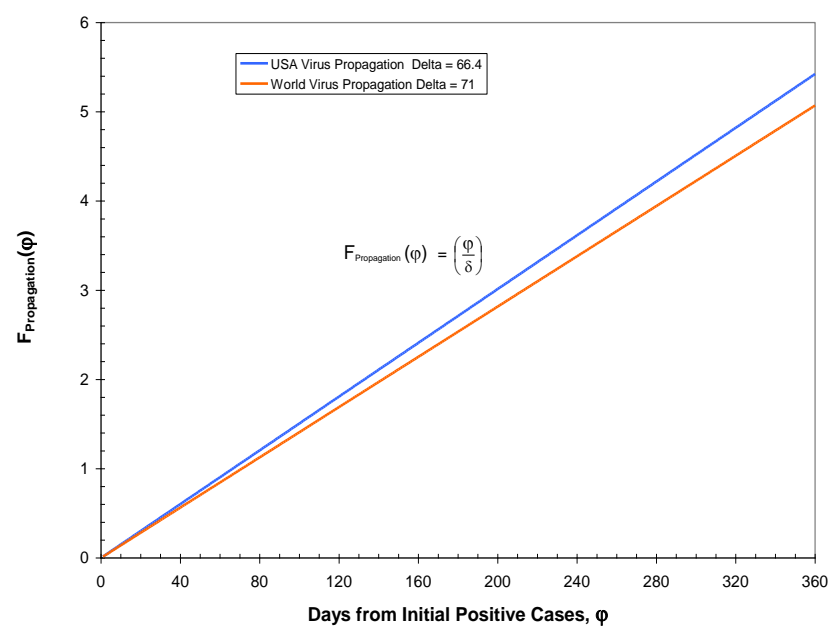

Fig. 7. Spreading Function, $F(\varphi)$, Comparison of Propagation Components $\mathrm{F}_{\text {Propagation }}(\varphi)$ for USA and World Data as of April 27, 2020.

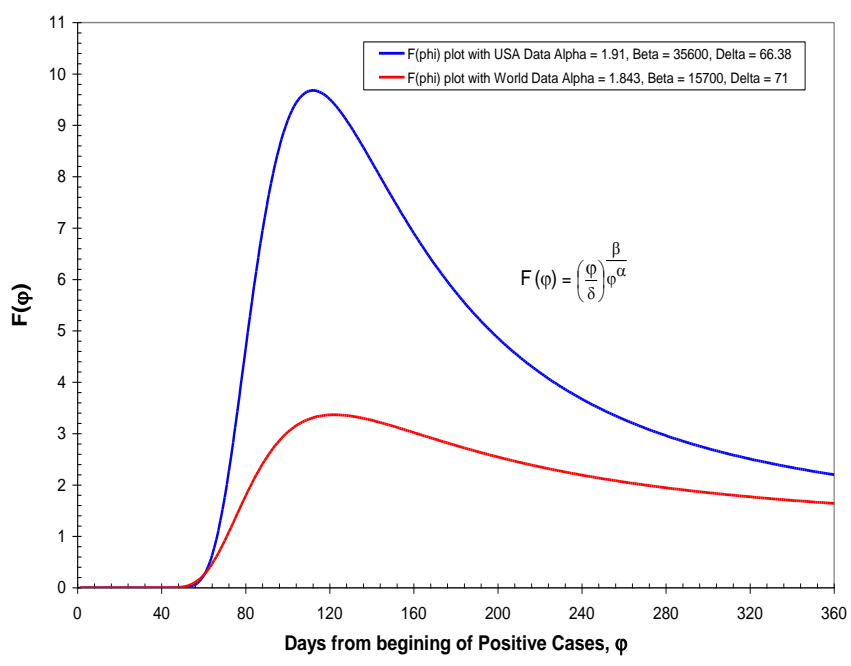




\section{Advanced Materials Letters www.vbripress.com/aml}

Fig. 8. Comparison of Spreading Function, $F(\varphi)$, plots for USA and World Corona COVID-19 Virus Data as of April 27, 2020.

The significant results indicated in Table 2 are clearly confirmed in Table 3. The results in Table $\mathbf{3}$ were again obtained using the data from Johns Hopkins University [18] as reported on CNN on April 30, 2020 [20]. The values in Table 3 for the total population in the USA [21] (as of August 28, 2019) as well as the World population [22] (as of June 2019) were obtained from the Web.

Note in Table 3 that the USA had $32.39 \%$ of the Worldwide positive cases of Corona Virus (COVID-19) while the USA had only $4.35 \%$ of the World population as of April 30, 2020. In addition, even though the death rate amongst infected people in the USA was only $5.86 \%$ compared to $7.11 \%$ Worldwide it was found that the USA had $26.72 \%$ of all the deaths from the Corona Virus (COVID-19) Worldwide.

Table 2. USA and World Spreading Function, $F(\varphi)$, Values for Corona Virus COVID-19 Data.

\begin{tabular}{lcc}
\hline & $\begin{array}{c}\text { Corona } \\
\text { Virus Model } \\
\text { for USA }\end{array}$ & $\begin{array}{c}\text { Corona } \\
\text { Virus Model } \\
\text { for World }\end{array}$ \\
\hline Mitigation Effectiveness, $\alpha$ & 1.910 & 1.843 \\
Mitigation Magnitude, $\beta$ & 35600 & 15700 \\
Threshold Upper Limit, $\delta$ & 66.4 & 71.0 \\
Final Positive Cases, $\mathrm{F}(\varphi)($ Limit $)$ & 1 & 1 \\
Days to Percolation Threshold, $\varphi_{\mathrm{c}}$ & $65(3 / 26 / 20)$ & $59.8(3 / 20 / 20)$ \\
Days to Early Inflection Point, $\varphi_{\mathrm{EIP}}$ & $79(4 / 8 / 20)$ & $75(4 / 4 / 20)$ \\
Days to Maximum Positive Cases, $\varphi_{\text {Max }}$ & 112.1 & 122.2 \\
& $(5 / 12 / 20)$ & $(5 / 21 / 20)$ \\
Days to Later Inflection Point, $\varphi_{\mathrm{LIP}}$ & $143(6 / 11 / 20)$ & $163(7 / 1 / 20)$ \\
Maximum Slope, $(\mathrm{dF}(\varphi) / \mathrm{d} \varphi)_{\text {Max }}$ & 0.3110 & 0.0891 \\
Positive Cases, $\mathrm{F}(\varphi)$ at $\varphi_{\text {Max }}$ & 9.680 & 3.367 \\
Positive Cases, $\mathrm{F}(\varphi)$ at 360 Days & 2.201 & 1.641 \\
\hline
\end{tabular}

Consequently, the results in Table 3 confirm the alarming results shown in Fig. 8 and Table 2 that the epidemic of the Corona Virus (COVID-19) in the USA appears to be best described as being out of control compared to the rest of the World.

Table 3. USA and World Data for Positive Cases of Corona Virus (As of April 30, 2020 - CNN).

\begin{tabular}{lcc}
\hline & USA & World \\
\hline Positive Cases & $1,040,488$ & $3,212,262$ \\
Deaths & 60,999 & 228,299 \\
\% Deaths & 5.86 & 7.11 \\
\% USA & 32.39 & \\
$\begin{array}{l}\text { Positive Cases } \\
\text { in World }\end{array}$ & & \\
\% USA Deaths & 26.72 & \\
in World & & \\
Population & $329,524,838$ & 7,577,130,400 \\
& (As of August & (Wune 2019) \\
& 28, 2019) & (Worldpopulationreview.com) \\
& & \\
\hline
\end{tabular}
$\%$ USA of
World
Population

\section{Discussion}

The new model describing the spread of disease in a population during an epidemic was successfully introduced in this study. The new disease spreading model was developed from a previously published model by this author that involved the prediction of the percolation threshold for electrical conducting composites. The Corona Virus data evaluated with this model was collected by Johns Hopkins University. One major advantage of this model is that it predicts where the maximum will be by utilizing data from very beginning of accumulation of positive cases. This new model also predicts the inflection points both before and after the maximum. This model is also able to separate the propagation of the disease from the mitigation of the disease. This information could be very helpful when fighting an epidemic.

The model developed in this study involved the cumulative confirmed infected people (C) or positive cases for the Corona Virus (COVID-19), the Infected number of people - at any point in time (I), Recovered number of people (R), and the people that have died from the virus (D). While this Coronavirus epidemic did presumably start with $I_{0}=0, R_{o}=0, D_{0}=0$, it is clear that the bell-shaped curve cannot effectively describe the spread of this virus. Unfortunately, those who have died or who have become permanently infected by the virus can not be accounted for with the bell-shaped curve. When the COVID-19 epidemic is finally under control it is virtually certain that a significant number of people will not be able to recover.

In general, it has also been found that this model has three concurrent mathematical conditions have been found to occur at the same curve location for $\mathrm{S}$-shaped curves with percolation thresholds. In particular, the maximum slope occurs at the first inflection point on the curve and the maximum slope at that same location is then used to calculate the straight line to obtain the maximum extrapolated percolation threshold on the $\mathrm{x}$-axis. Mathematically the inflection points were generated by setting second derivative of the model equal to zero.

The model introduced in this study consists of two parts: a magnitude component and a dimensionless $F(\varphi)$ component that allows different populations to be compared directly independent of their magnitude. Consequently, the accumulation of positive cases as described by this new model is simply a function that begins at $\varphi=0$ where $\mathrm{F}(\varphi)=0$ and $\sigma_{\mathrm{i}}=0$. However as $\varphi$ $\rightarrow \infty$ then $\mathrm{F}(\varphi) \rightarrow 1.0$ and $\sigma_{\mathrm{i}} \rightarrow \sigma_{\mathrm{F}}$ which yields a limit that is not zero and is not negligible.

In addition, the Spreading Function, $F(\varphi)$, can also be separated into a Propagation Component and a Mitigation Component. The Propagation Component, $F_{\text {Propagation }}(\varphi)$, is a straight line with slope $(1 / \delta)$ that increases linearly with the number of days, $\varphi$, since the first positive case. 


\section{Advanced Materials Letters www.vbripress.com/aml}

However, the Mitigation Component, $F_{\text {Mitigation }}(\varphi)$, decreases exponentially with an increase in the number of days, $\varphi$, since the first positive case. Note that as long as the number of days, $\varphi$, are less than the number of days as indicated by the Threshold Upper Limit, $\delta$, then the Propagation Component will be a fraction less than 1 . This means that as long as the Mitigation Component, $\mathrm{F}_{\text {Mitigation }}$ $(\varphi)$, is a large number as well as an exponent for the Propagation Component, FPropagation $(\varphi)$, then the combination described as Spreading Function, $F(\varphi)$, will generate a very small number essentially approaching zero when $\varphi$ is less than $\delta$. However, once the number of days, $\varphi$, exceeds the value of $\delta$ then the Propagation Component, $\mathrm{F}$ Propagation $(\varphi)$, will no longer be a fraction less than 1 and the Propagation Component will continue to increase significantly while the Mitigation Component will more rapidly approach zero and forcing the combination of the two to cause the function $F(\varphi)$ to approach the value of one.

The Corona Virus (COVID-19) data used in this study for both the USA and the World were collected and reported by Johns Hopkins University on their website [18]. However, only the data between January 22 and April 27 were utilized in this study. Since there seemed to be a general disregard for "Social Distancing" after April 27, it was felt that the best data for evaluating the model introduced in this study occurred in that time frame.

Initially it did appear that it would be difficult to compare the World and USA Corona Virus (COVID-19) data directly. However, it was found that these data sets are really much easier to compare directly using their $F(\varphi)$ components independent of the magnitude of their final projected Positive Corona Virus Cases, $\sigma \mathrm{F}$. A direct comparison of the $\mathrm{F}(\varphi)$ components for both the USA and the World Corona Virus data results was found to yield significant new insights into a comparison these sets of data.

\section{Conclusions}

In general it was found that the comparison of the Spreading Function, $F(\varphi)$, component results suggested that both the Mitigation and the Propagation process appeared to be somewhat more effective in the USA than in the World. Unfortunately, these counteracting results did not directly clarify which component was more dominant.

However, when the magnitude of the separate populations was eliminated it was apparent that the growth of the epidemic in the USA was significantly greater than that experienced by the World data. When the magnitude of the separate populations was neutralized, then the Spreading Function, $F(\varphi)$, values clearly showed that the maximum rate of growth of the Corona Virus epidemic at their initial inflection points was significantly greater for the USA compared to the World data. In addition, when the magnitude of the separate populations was neutralized, then the results clearly showed that the USA achieved a significantly greater relative maximum value than the World data. Finally, after 360 days the $F(\varphi)$ results indicated that the USA will still have a higher relative level of people infected by the Corona Virus compared to the World data.

These significant results were supported with the finding that the USA had $32.39 \%$ of the Worldwide positive cases of Corona Virus (COVID-19) while the USA had only $4.35 \%$ of the World population as of April 30,2020 . In addition, even though the death rate amongst infected people in the USA was only $5.86 \%$ compared to $7.11 \%$ Worldwide it was found that the USA had $26.72 \%$ of all the deaths Worldwide as of April 30.

Consequently, this study confirmed the alarming indication that the Corona Virus (COVID-19) in the USA appears be best described as being out of control.

\section{Appendix A - Derivatives for the New Corona Virus (COVID-19) Model}

The Corona Virus model is:

$$
\sigma_{i}=\sigma_{F}\left(\frac{\varphi}{\delta}\right)^{\frac{\beta}{\varphi^{\alpha}}}
$$

The first derivative of equation (A-1) gives

$$
\begin{aligned}
\frac{d \sigma_{i}}{d \varphi} & =\sigma F\left(\frac{\beta}{(\varphi)^{\alpha+1}}\right)\left(\frac{\varphi}{\delta}\right) \frac{\beta}{\varphi^{\alpha}}\left(1-\alpha \operatorname{Ln}\left(\frac{\varphi}{\delta}\right)\right) \\
\frac{d \sigma_{i}}{d \varphi} & =\sigma_{i}\left(\frac{\beta}{(\varphi)^{\alpha+1}}\right)\left(1-\alpha \operatorname{Ln}\left(\frac{\varphi}{\delta}\right)\right)
\end{aligned}
$$

Note that $\frac{d \sigma_{i}}{d \varphi}=0$ when

$$
\left(1-\alpha \operatorname{Ln}\left(\frac{\varphi}{\delta}\right)\right)=0
$$

Solving equation (A-4) gives the maximum at

$$
\begin{aligned}
\varphi_{\max } & =\delta \mathrm{e}^{(1 / \alpha)} \\
& \text { If } \mathrm{F}(\varphi)=\left(\frac{\varphi}{\delta}\right)^{\frac{\beta}{\varphi^{\alpha}}}
\end{aligned}
$$

then at $\varphi_{\max }$

$$
\mathrm{F}\left(\varphi_{\max }\right)=\left(\mathrm{e}^{1 / \alpha}\right)^{\beta / \mathrm{e}_{\delta}^{\alpha}}
$$

The second derivative of the percolation threshold model (A-1) gives

$$
\begin{aligned}
& \frac{d^{2} \sigma_{i}}{d \varphi^{2}}=\sigma F\left(\frac{\beta}{(\varphi)^{\alpha+2}}\right)\left(\frac{\varphi}{\delta}\right)^{\frac{\beta}{\varphi^{\alpha}}}\left(z^{2}\left(\frac{\beta}{(\varphi)^{\alpha}}\right)-z(\alpha+1)-\alpha\right)^{(A-} \\
& \frac{d^{2} \sigma_{i}}{d \varphi^{2}}=\sigma_{i}\left(\frac{\beta}{(\varphi)^{\alpha+2}}\right)\left(z^{2}\left(\frac{\beta}{(\varphi)^{\alpha}}\right)-z(\alpha+1)-\alpha\right)
\end{aligned}
$$




$$
z=\left(1-\alpha \operatorname{Ln}\left(\frac{\varphi}{\delta}\right)\right)
$$

At an inflection point $\frac{d^{2} \sigma_{i}}{d \varphi^{2}}=0$

An inflection point can also be identified by trial and error when

$$
\left(z^{2}\left(\frac{\beta}{(\varphi)^{\alpha}}\right)-z(\alpha+1)-\alpha\right)=0
$$

Appendix B - An Optional Approach to Calculate the Percolation Threshold Using the New Model to Address a Disease Epidemic

For a straight line through the point at $\varphi$ the slope can be calculated using equation (A-3) as

$$
(\operatorname{Slope}(\varphi))=\frac{d \sigma_{i}}{d \varphi}=\sigma_{i}\left(\frac{\beta}{(\varphi)^{\alpha+1}}\right)\left(1-\alpha \operatorname{Ln}\left(\frac{\varphi}{\delta}\right)\right)
$$

Then the straight line through the point at $\varphi$ will have the following equation.

$$
\sigma_{\mathrm{i}}=(\operatorname{Slope}(\varphi)) \varphi+(\operatorname{Intercept}(\varphi))
$$

When $\sigma_{\mathrm{I}}=0$, then the Projected Threshold, $\varphi_{\mathrm{ci}}$, on the concentration axis can then be calculated at each point as

$$
\begin{aligned}
& \varphi_{C_{\mathrm{i}}}=-\frac{(\text { Intercept }(\varphi))}{(\operatorname{Slope}(\varphi))}=\frac{(\operatorname{Slope}(\varphi)) \varphi-\sigma_{\mathrm{i}}}{(\operatorname{Slope}(\varphi))}=\varphi-\frac{\sigma_{\mathrm{i}}}{(\text { Slope }(\varphi))} \\
& \varphi_{\mathrm{ci}}=\varphi-\left(\frac{1}{\beta}\right)\left(\frac{\varphi^{\alpha+1}}{1-\alpha \operatorname{Ln}\left(\frac{\varphi}{\delta}\right)}\right)
\end{aligned}
$$

The maximum value of $\varphi_{\mathrm{ci}}$ calculated by equation (B-4) between $\varphi=0$ and $\varphi_{\max }=\delta \mathrm{e}^{(1 / \alpha)}$ then yields the desired Percolation Threshold, $\varphi_{c}=\left(\varphi_{\mathrm{ci}}\right)_{\max }$.

\section{Conflict of interest statement}

This research did not receive any specific grant from funding agencies in the public, commercial, or not-for-profit sectors. Consequently, the author is not aware of any conflict of interest considerations in association with the publication of this article.

\section{Keywords}

Corona Virus (COVID-19), percolation threshold, disease spread, epidemic, positive cases.

\section{Received: 11 December 2020 \\ Revised: 31 January 2021 \\ Accepted: 13 February 2021}

\section{References}

1. Fanelli, D.; Piazza, F.; Chaos Solitons \& Fractals, 2020, 134.

2. Wang, H.W.; Wang, Z.Z.; Dong, Y.Q.; Chang, R.J.; Xu, C. et al.; Cell Discovery, 2020, 6.

3. Roosa, K.; Lee, Y.; Luo, R.; Kirpich, A. et al.; Journal of Clinical Medicine, 2020, 9

4. Yang, C.Y.; Wang, J.; Mathematical Biosciences and Engineering, 2020, 17, 2708.
5. Chang, R.J.; Wang, H.W.; Zhang, S.X.; Wang, Z.Z. et al.; Frontiers of Medicine, 2020.

6. Gilbert, M.; Pullano, G.; Pinotti, F.; Valdano, E. et al.: Lancet, 2020 395, 871.

7. Yang, Z.F.; Zeng, Z.Q.; Wang, K.; Wong, S.S. et al.; Journal of Thoracic Disease, 2020, 12, 165.

8. Jia, J.W.; Ding, J.; Liu, S.Y.; Liao, G.D. et al.; Electronic Journal of Differential Equations, 2020, 23.

9. Gilbert, M.; Pullano, G.; Pinotti, F.; Valdano, E. et al.; Lancet, 2020, 395, 871.

10. Kim, S.; Kim, Y.J.; Peck, K.R.; Jung, E.; Journal of Korean Medical Science, 2020, 35.

11. Moftakhar, L.; Seif, M.; Archives of Iranian Medicine, 2020, 23, 235.

12. Zareie, B.; Roshani, A.; Mansournia, M.A.; Rasouli, M.A.; Moradi, G.; Archives of Iranian Medicine, 2020, 23, 244.

13. Wang, Y.X.; Wang, Y.Y.; Chen, Y.; Qin, Q., Journal of Medical Virology, 2020, 92, 568.

14. Zhao, S.; Stone, L.; Gao, D.; Musa, S.S.; Annals of Translational Medicine, 2020, 8 .

15. Zhao, S.; Stone, L.; Gao, D.Z.; Musa, S.S.; Chong, MKC et al.; Annals of Translational Medicine, 2020, 8 .

16. Squazzoni, F.; Polhill, J.G.; Edmonds, B.; Ahrweiler, P. et al.; JASSS - The Journal of Artificial Societies and Social Simulation, 2020, 23

17. Bai, Z.H.; Gong, Y.; Tian, X.D.; Cao, Y. et al.; Virologica Sinica 2020.

18. Gardner, L.; Department of Civil and Systems Engineering, Johns Hopkins University Website, https://www.arcgis.com/apps/ opsdashboard/index.html\#/bda7594740fd40299423467b48e9ecf6, April 27, 2020.

19. Sudduth, R.D.; Journal of Applied Polymer Science, 2019, 136, 1.

20. Data from Johns Hopkins University as reported on the television station CNN on April 30, 2020.

21. Population of the United States of America as of August 28, 2019 as reported on Wikipedia web site https://en.wikipedia.org/wiki/ Demographics_of_the_United_States (April 30, 2020).

22. World Population as of June 2019 as reported on the website, Worldpopulationreview.com (April 30, 2020). 